\title{
Membrane type-1 matrix metalloproteinase and TIMP-2 in tumor angiogenesis
}

\author{
N.E. Sounni, M. Janssen, J.M. Foidart, A. Noel \\ Laboratory of Tumor and Development Biology, University of Liège, Sart Tilman, B-4000 Liège, Belgium
}

\begin{abstract}
The matrix metalloproteinases (MMPs) constitute a multigene family of over 23 secreted and cell-surface associated enzymes that cleave or degrade various pericellular substrates. In addition to virtually all extracellular matrix (ECM) compounds, their targets include other proteinases, chemotactic molecules, latent growth factors, growth factor-binding proteins and cell surface molecules. The MMP activity is controlled by the physiological tissue inhibitors of MMPs (TIMPs). There is much evidence that MMPs and their inhibitors play a key role during extracellular remodeling in physiological situations and in cancer progression. They have other functions that promoting tumor invasion. Indeed, they regulate early stages of tumor progression such as tumor growth and angiogenesis. Membrane type MMPs (MT-MMPs) constitute a new subset of cell surface-associated MMPs. The present review will focus on MT1-MMP which plays a major role at least, in the ECM remodeling, directly by degrading several of its components, and indirectly by activating pro-MMP2. As our knowledge on the field of MT1-MMP biology has grown, the unforeseen complexities of this enzyme and its interaction with its inhibitor TIMP-2 have emerged, often revealing unexpected mechanisms of action.
\end{abstract}

Keywords: MT1-MMP ; tumoral angiogenesis ; vascular endothelial growth factor ; protease inhibitors ; estradiol

\section{MMP structure and functions}

Matrix metalloproteinases (MMPs) are a broad family of zinc-binding endopeptidases that play a key role in the extracellular matrix (ECM) degradation associated with cancer cell invasion, metastasis and angiogenesis (Egeblad and Werb, 2002; McCawley and Matrisian, 2000). At present, more than 21 human MMPs and the homologues from other species have been identified (Egeblad and Werb, 2002). The MMP family can be segregated into two groups, the soluble type and the membrane-type MMPs (MT-MMPs and MMP23). Although initially classified according to their substrate specificity (McCawley and Matrisian, 2000), the MMP classification is now based on their structure (Egeblad and Werb, 2002). The 'minimal-domain MMPs' (MMP7 and MMP26) contains (i) a signal peptide that directs them to the endoplasmic reticulum, (ii) a propeptide, with a zinc-interacting thiol (SH) group that maintains the proMMP in a latent form (zymogen), and (iii) a catalytic domain containing the highly conserved $\mathrm{Zn}^{2+}$ binding site (HEXGHXXGXXHS/T) (Fig. 1). With the exception of MMP23, all other MMPs display a prolinerich hinge region that links the catalytic domain to the hemopexin/vitronectin-like domain. This C-terminal domain influences substrate specificity and the binding to tissue inhibitors of MMPs (TIMPs) and cell surface molecules. The hemopexin-containing MMPs are further distinguished by the presence of specific insert(s). The gelatin-binding MMPs (MMP2 and MMP9 or gelatinases) contain inserts that resemble the collagen-binding type II repeats of fibronectin. The membrane type (MT)-MMPs have a single pass transmembrane domain and a short cytoplasmic tail (MMP14, 15, 16 and 24), or a hydrophobic region that acts as a glycosyl phosphatidylinositol (GPI)-membrane anchoring signal (MMP17 and MMP25). A recognition motif for intracellular furin-like serine proteinase is inserted between the pro- and catalytic domains of furin-activated secreted MMPs (MMP11 and MMP28). This motif is also found in the 'vitronectin-like insert (VN) MMP' (MMP21) and MT-MMPs. Finally, MMP23 has unique cystein-rich, prolinerich and IL-1 type II receptor-like domains instead of a hemopexin domain (Pei et al., 2000).

Initially, MMPs were thought to predominantly degrade ECM components. By this way, MMPs were believed to promote cell migration, affect cell functions and modulate growth factor bioavailability (Bergers et al., 2000). More recently, MMPs have also been implicated in the cleavage of chemokines (McQuibban et al., 2001), growth factor receptors or growth factor binding proteins, the activation of growth factors, the release of cell membrane-bound precursor forms of growth factors and the cleavage of cell adhesion molecules such as cadherin and CD44 (reviewed in Egeblad and Werb, 2002).

Due to the overlapping of MMP substrate specificities, the function of individual proteases is dictated by their differential expression patterns. To accomplish their functions, MMPs must be present at the right time, at the right place (extracellular or pericellular location) and under appropriate inhibited or activated form. Therefore, MMPs are tightly regulated at the transcriptional and posttranscriptional levels, as well as at the protein levels 
via their activators, inhibitors and cell surface localization (reviewed in Sternlicht and Werb, 2001). Most of the MMPs are activated outside the cell by other MMPs or serine proteinases. However, MMP11, MMP28 and MTMMPs can be activated intracellularly, before they reach the cell surface by furin-like serine proteinases (for review, see Egeblad and Werb, 2002; Sternlicht and Werb, 2001). More recently, the shedding of MT-MMP appears as an additional way to control enzyme localization and activity (Wang and Pei, 2001). MMP activity is tightly controlled by endogenous inhibitors such as $\alpha 2$-macroglobulin and specific MMP inhibitors, the TIMPs. TIMPs-1, -2, -3, -4 reversibly inhibit MMPs in a 1:1 stoichiometric fashion (Sternlicht and Werb, 2001).

The different TIMPs differ in tissue-specific expression and ability to inhibit various MMPs and their capacity to interact or not with pro-MMPs (Jiang et al., 2002; Sternlicht and Werb, 2001).

Fig. 1. Domain structure of the MMPs. Pre, signal sequence; Pro, propeptide; Fu, furin-like serine proteinases recognition site; $\mathrm{Zn}$, zinc-binding site; Fi, fibronectin-like collagen-binding type II inserts; $H$, hinge region; Vn, vitronectin-like insert; TM, transmembrane domain; Cy, cytoplasmic tail; GPI, glycosyl phosphatidylinositol-anchoring domain; CA cysteine array domain; Ig-like, immunoglobulin-like domain. See description in the text.

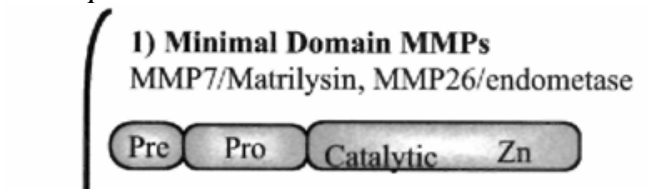

2) Simple hemopexin domain-containing MMPs

MMP1/collagenase-1, MMP8/collagenase-2, MMP13/collagenase-3,

MMP18/collagenase-4, MMP3/stromelysin-1, MMP10/stromelysin-2, MMP27,

MMP12/metalloelastase, MMP19/RASI-1, MMP20/enamelysin, MMP22/CMMP

Pre Pro Catalytic $\mathrm{Zn}$ Hemopexin

3) Gelatin-binding MMPs

MMP2/gelatinase A, MMP9/gelatinase B

Pre Pro Catalytic (Fi) Fi/Fi) $\mathrm{Zn}$ Hemopexin

4) Furin-activated secreted MMPs

MMP11/stromelysin-3, MMP28/epilysin

Pre Pro Eu Catalytic $\mathrm{Zn}$ Hemopexin

5) Vitronectin-like insert MMPs

MMP21/homomologue of Xenopus XMMP

Pre Pro Eu Catalytic (Vn) $\mathrm{Zn}$ Hemopexin

6) Transmembrane MMPs

MMP14/MT1-MMP, MMP15/MT2-MMP, MMP16/MT3-MMP,

MMP24/MT5-MMP

Pre Pro Fu Catalytic $\mathrm{Zn}$ Hemopexin (TM) (Cy

7) GPI-anchored MMPs

MMP17/MT4-MMP, MMP25/MT6-MMP

Pre Pro Fu Catalytic $\mathrm{Zn}$ Hemopexin (GPI)

8) Type II transmembrane MMPs

MMP23/cysteine array MMP

Pre Pro Fu Catalytic $\mathrm{Zn}$ CA Ig-like




\section{Membrane-associated MMPs}

Even though the majority of MMPs are secreted as soluble enzymes into the extracellular milieu, a growing list of newly identified MMPs are membrane-bound by at least three distinct anchoring mechanisms (Fig. 1): (i) type I transmembrane domain for MT1, -2, -3 and -5; (ii) GPI linkage for MT4 and -6 MMPs; and (iii) type II transmembrane domain for MMP23/cystein array MMP. MT1-MMP (MMP14) is the prototypic member of the MT-MMPs and its expression has been associated with a variety of cellular and developmental processes, as well as multiple pathophysiological conditions (Pepper, 2001; Yana and Seiki, 2002). MT1-MMP displays a broad spectrum of activity against ECM components such as type I and II collagen, fibronectin, vitronectin, laminin, fibrin and proteoglycan (d'Ortho et al., 1997; Koshikawa et al., 2000). However, it has substrates that extend beyond ECM components such as cell surface molecules including CD44 (Kajita et al., 2001), pro $\alpha_{V}$ integrin (Deryugina et al., 2002b) and transglutaminase (Belkin et al., 2001). It interacts with integrins (Deryugina et al., 2001) and participates in the $\alpha_{\mathrm{V}} \beta_{8}$-mediated activation of TGF $\beta$ (Mu et al., 2002).

Perhaps, the most interesting aspect of MT1-MMP is the nature of its interactions with TIMP-2 and the role that they play together in pro-MMP2 activation. A model for the activation of pro-MMP2 has been proposed in which the catalytic domain of MT1-MMP binds to the N-terminal portion of TIMP-2, leaving the TIMP-2 Cterminal region available for binding to the hemopexin-like domain of pro-MMP2 (Fig. 2) (Sternlicht and Werb, 2001). This ternary complex has been suggested to cluster pro-MMP2 at the cell surface near a TIMP-2-free MT1-MMP molecule which is thought to initiate the activation of the bound pro-MMP2. The MT1-MMP oligomerization may facilitate this process of pro-MMP2 activation (Itoh et al., 2001; Lehti et al., 2002). According to this model (Fig. 2), pro-MMP2 activation would occur only at low concentration of TIMP-2 relative to MT1-MMP, allowing availability of active MT1-MMP to activate pro-MMP2 bound in the ternary complex. The absolute dependence of this activation pathway on the presence of TIMP-2 has been demonstrated in vivo by using TIMP-2 deficient mice (Wang et al., 2000). MT1-MMP appears to be the major activator of MMP2 as shown by generating MT1-MMP deficient mice (Zhou et al., 2000). However, in these mice, some activation of MMP2 was observed which may be related to the action of other MT-MMPs. Indeed, MT-MMP-2, $-3,-5,-6$ have been shown to activate pro-MMP2, using either catalytic domains in vitro or full-length molecules (MT-MMP-3, -5, -6) (Kolkenbrock et al., 1997; Pei, 1999; Takino et al., 1995; Llano et al., 1999; Velasco et al., 2000). However, the requirement for TIMP-2 in pro-MMP2 activation by MT-MMPs 2-6 is less well known. Recently, Morrison et al. (2001) reported that MT2-MMP efficiently activates MMP2 in a TIMP-2-inde-pendent pathway.

Fig. 2. Model of pro-MMP2 activation by MT1-MMP. (1) pro-MT1-MMP can be activated intracellularly by furin or extracellularly by plasmin. (2) TIMP-2 acts as an adaptor molecule and bridges MT1-MMP to proMMP2. A second TIMP-2-free molecule cleaves a portion of the MMP2 prodomain leading to the formation of an intermediate MMP2 form. (3) Further cleavage of MMP2 involving an autocatalytic process or the cooperation of plasmin results in the final maturation and release of active MMP2 from the cell surface. (4) MT1-MMP and TIMP-2 are then internalized in the cytoplasm. Nt, N-terminal part and Ct, C-terminal portion of TIMP-2.

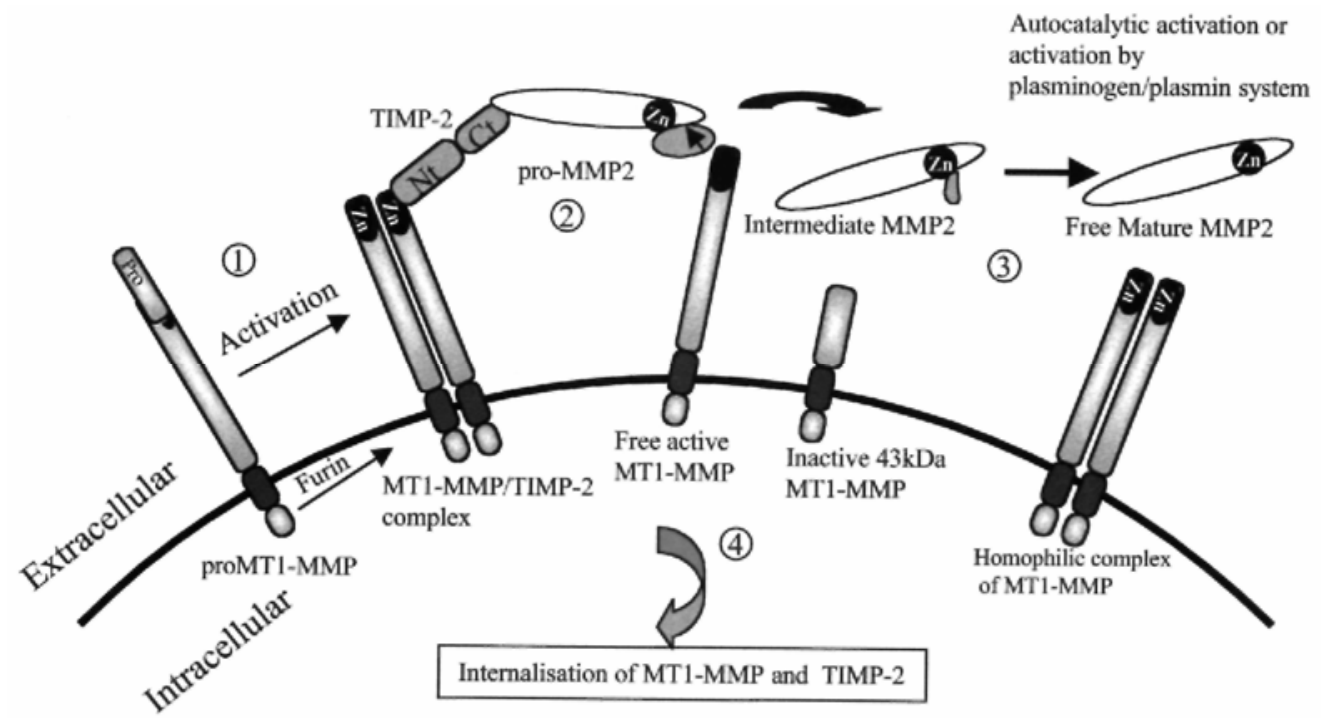




\section{MT1-MMP and cancer}

The expression of MT1-MMP has been reported to correlate with the malignancy of different tumor types, such as lung (Nawrocki et al., 1997), gastric (Mori et al., 1997), colon, breast carcinomas (Okada et al., 1995), gliomas (Belien et al., 1999) and melanomas (Hofmann et al., 2000). An important issue during the last 10 years was to determine the cellular source of MMPs involved during cancer progression. For instance, studies of breast adenocarcinoma have revealed that stromal cells are strongly involved in the MMP production. Thus, mRNAs for MMP-1, MMP2 (Polette et al., 1997), MMP-11 (Basset et al., 1990), MMP-13 (Nielsen et al., 2001) and MT1-MMP (Okada et al., 1995) have been all found to be expressed by fibroblast-like cells. The fibroblastic cells expressing MMP-11 (Unden et al., 1996), MMP-13 (Nielsen et al., 2001) or MT1-MMP (unpublished data) have been identified as myofibroblasts. It is particularly noteworthy that cocultivation of fibroblasts with tumor cells induced the up-regulation of MMP2 and MT1-MMP expression by fibroblasts (Noel et al., 1994; Polette et al., 1997). Cancer cells might enhance stromal cells to produce MMPs in a paracrine manner through secretion of cytokines, growth factors or EMMPRIN (Sternlicht and Werb, 2001). These findings are consistent with the observation that fibroblasts promoted tumor progression in animal models through their production of MMPs (Masson et al., 1998; Noel et al., 1993) and emphasize the importance of tumor-host interactions during cancer progression. MT1-MMP is also expressed by endothelial cells during migration (Galvez et al., 2001; Hiraoka et al., 1998).

MT1-MMP appears more and more has a complex multifunctional molecule influencing different cell functions (Fig. 4). Its importance during development is supported by the severe phenotype associated with MT1-MMP deficiency in mice (Holmbeck et al., 1999; Zhou et al., 2000). Indeed, MT1-MMP-deficient mice exhibit damages in skeletal development manifested by craniofacial dysmorphism, dwarfism, osteopenia and fibrosis (Holmbeck et al., 1999; Zhou et al., 2000). The expression of MT1-MMP has been described in processes involving cell migration (Koshikawa et al., 2000). Overexpression of MT1-MMP increases the number of experimental metastases (Tsunezuka et al., 1996). The generation of a permissive substrate for cell migration via the degradation of various specific ECM components is considered as a key event facilitating cell migration. MT1-MMP may function at least as a fibrinolytic and gelatinolytic enzyme (Hotary et al., 2000; d'Ortho et al., 1997). Cleavage of laminin-5 by MMP2 and MT1-MMP reveals a cryptic site that trigger epithelial cell motility (Gilles et al., 2001; Koshikawa et al., 2000). However, recent data indicate that MMPs and particularly MT1MMP do far more to promote cancer progression than merely remove the physical barriers to migration and invasion (Egeblad and Werb, 2002; McCawley and Matrisian, 2000). MT1-MMP acts as a processing enzyme for CD44 (the main receptor for hualuronan), releasing it as a soluble fragment in the medium and stimulating cell motility (Kajita et al., 2001). Cleavage of CD44 by MT1-MMP is likely to also downregulate MMP9 cellassociated activity (Yu and Stamenkovic, 1999).

\section{Role of MT1-MMP during tumoral angiogenesis}

Although it has been previously reported that MT1-MMP can participate in developmental and physiological angiogenesis (Holmbeck et al., 1999; Zhou et al., 2000), its role during tumor angiogenesis has been only recently established. We have previously demonstrated that the overexpression of MT1-MMP in human melanoma A2058 cells which produced only pro-MMP2 was associated with enhanced in vitro invasion and increased in vivo tumor growth and vascularization (Sounni et al., 2002a). We also investigated the effects of MT1-MMP overexpression on in vitro and in vivo properties of human breast adenocarcinoma MCF7 cells which do not express either MT1-MMP, or MMP2. MT1-MMP and MMP2 cDNAs were either transfected alone or cotransfected (Sounni et al., 2002b). All clones overexpressing MT1-MMP (i) were able to activate endogenous or exogenous pro-MMP2, (ii) displayed an enhanced in vitro invasiveness through matrigel-coated filters, independently of MMP2 transfection, (iii) induced the rapid development of highly vascularized tumors when injected subcutanously in nude mice, and (iv) promoted blood vessels sprouting in the rat aortic ring assay, an in vitro model of angiogenesis (Sounni et al., 2002b). These data demonstrating, in two experimental models (Sounni et al., 2002a,b), the relation between MT1-MMP expression and an angiogenic phenotype are in accordance with the in vivo observations reported by other groups (Deryugina et al., 2002b). Since MCF7 cells are estrodiol-dependent cells, the tumorigenicity and angiogenic phenotype of MT1-MMP overexpressing clones were compared after inoculation into nude mice supplemented with estradiol pellets or into nude ovariectomized mice. The over-expression of MT1-MMP did not bypass the dependancy to estradiol (Fig. 3).

MT1-MMP may contribute to tumor angiogenesis through different mechanisms (Pepper, 2001). Recently, the MT1-MMP has been shown to interact with the $\alpha v \beta 3$ integrin which plays a major role during angiogenesis (Deryugina et al., 2001). However, the implication of $\alpha v \beta 3$ in the MCF7 cell system is unlikely since these cells do not express the $\beta 3$ integrin subunit. Alternatively, MT1-MMP may function as a fibrinolytic enzyme in the absence of plasmin and promote angiogenesis in fibrin matrix (Hotary et al., 2000). In addition to such control of cell migration by regulating pericellular proteolysis directly or indirectly by activating pro-MMP2, we have pointed out a new mechanism of action of MT1-MMP, the regulation at a transcriptional level of vascular 
endothelial growth factor (VEGF) expression (Sounni et al., 2002b). Indeed, an enhancement of VEGF at the mRNA and protein levels was concomitant with MT1-MMP overexpression in cultured cells, as well as in tumor extracts. Such a modulation of VEGF production by MT1-MMP is further supported by the study of Deryugina using glioma cells (Deryugina et al., 2002b). In contrast to MMP9 which modulates VEGF bioavailability by releasing it from the ECM (Bergers et al., 2000), MT1-MMP appears to control gene expression. Whether MT1MMP exerts this effect directly or indirectly remains to be elucidated. It is likely that MT1-MMP transduces an intracellular signal through its cytoplasmic C-terminal domain (Gingras et al., 2001) or interaction with integrin (Deryugina et al., 2002a). Interestingly, in an in vivo murine model, TIMP-2-mediated inhibition of tumor growth and angiogenesis was associated with a down-regulation of VEGF expression in tumor cells (Hajitou et al., 2001). All together, these results emphasize the key role played by TIMP-2 and MT1-MMP during tumoral angiogenesis.

Fig. 3. Growth curves of MCF7 tumors induced in ovariectomized nude mice. MCF7 cells overexpressing MT1MMP or control MCF7 cells were subcutaneously injected into ovariectomized nude mice supplemented or not with estradiol implants.

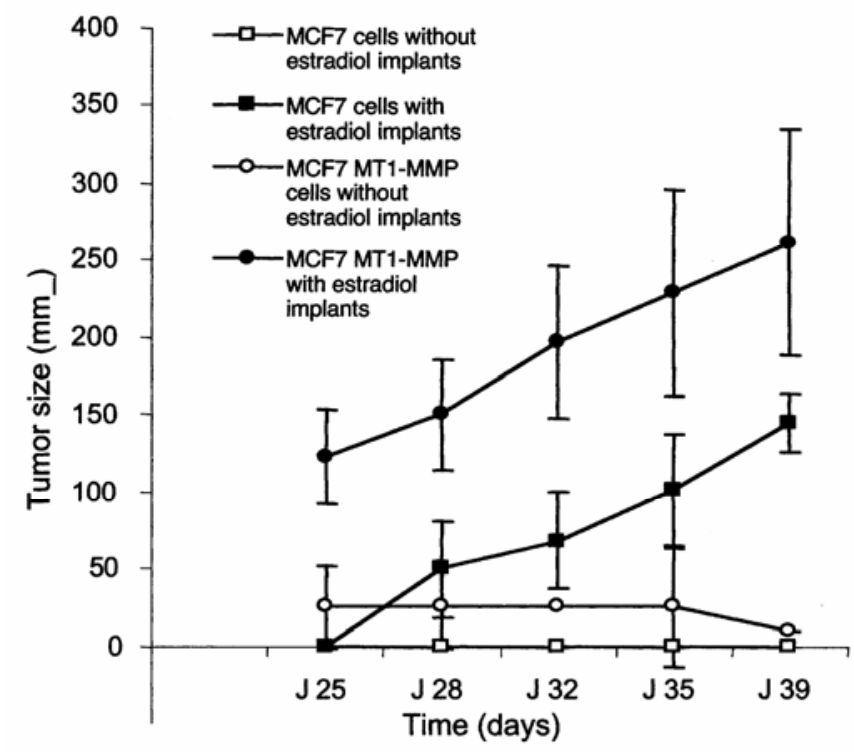

Fig. 4. Functions of MT1-MMP in cancer progression. MT1-MMP regulates cell migration, tumor growth and angiogenesis by different mechanisms: (1) MT1-MMP participates in pericellular proteolysis by acting directly against ECM components or indirectly by activating pro-MMP2. (2) MT1-MMP cleaves cell surface molecules, leading to their activation, inhibition or shedding. (3) MT1-MMP participates in signal transduction directly or by interacting with integrins.

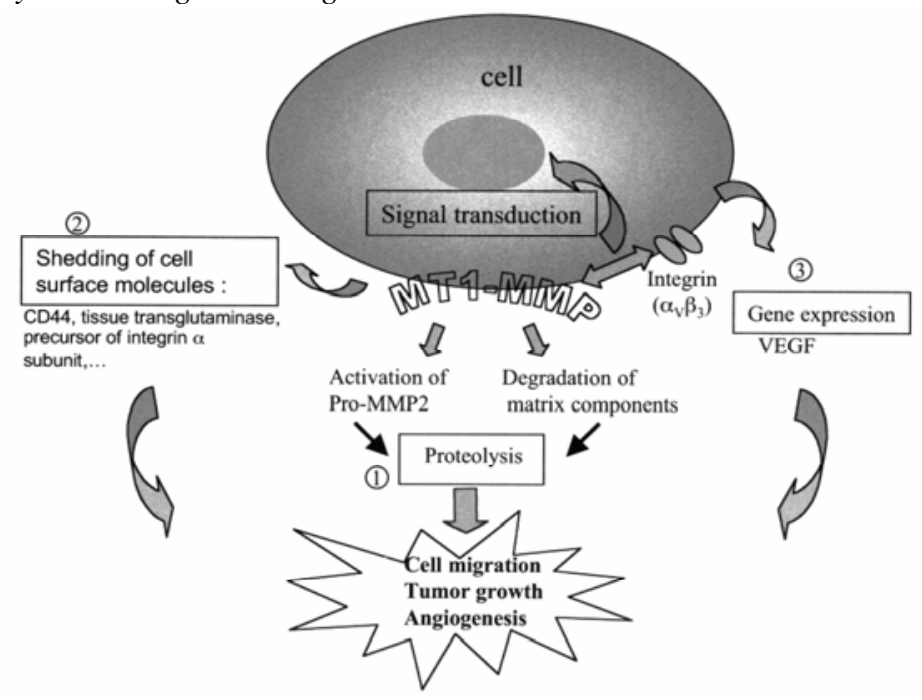


Published in: Matrix Biology (2003), vol. 22, iss. 1, pp. 55-61.

Status: Postprint (Author's version)

\section{Acknowledgments}

This work was supported by grants from the Communauté Française de Belgique (Actions de Recherches Concertées), the Commission of European Communities, the Fonds de la Recherche Scientifique Médicale, the Fonds National de la Recherche Scientifique (FNRS, Belgium), the Fédération Belge Contre le Cancer, the Fonds Spéciaux de la Recherche (University of Liège), the Centre Anticancéreux près l'Université de Liège, the FB Assurances, the Fondation Léon Frédéricq (University of Liège), the D.G.T.R.E. from the 'Région Wallonne', the Fonds d'Investissements de la Recherche Scientifique (CHU, Liège, Belgium).

\section{References}

Basset, P., Bellocq, J.P., Wolf, C., Stoll, I., Hutin, P., Limacher, J.M., Podhajcer, O.L., Chenard, M.P., Rio, M.C., Chambon, P., 1990. A novel metalloproteinase gene specifically expressed in stromal cells of breast carinomas. Nature 348, 699-704.

Belien, A.T., Paganetti, P.A., Schwab, M.E., 1999. Membrane-type 1 matrix metalloprotease (MT1-MMP) enables invasive migration of glioma cells in central nervous system white matter. J. Cell Biol. 144, 373-384.

Belkin, A.M., Akimov, S.S., Zaritskaya, L.S., Ratnikov, B.I., Deryugina, E.I., Strongin, A.Y., 2001. Matrix-dependent proteolysis of surface transglutaminase by membrane-type metalloproteinase regulates cancer cell adhesion and locomotion. J. Biol. Chem. 276, 18415-18422.

Bergers, G., Brekken, R., McMahon, G.A., et al., 2000. Matrix metalloproteinase-9 triggers the angiogenic switch during carcinogenesis. Nat. Cell Biol. 2, 737-744.

d'Ortho, M.P., Will, H., Atkinson, S., et al., 1997. Membrane-type matrix metalloproteinases 1 and 2 exhibit broad-spectrum proteolytic capacities comparable to many matrix metalloproteinases. Eur. J. Biochem. 250, 751-757.

Deryugina, E.I., Ratnikov, B., Monosov, E., et al., 2001. MT1-MMP initiates activation of pro-MMP2 and integrin alphavbeta3 promotes maturation of MMP2 in breast carcinoma cells. Exp. Cell Res. 263, 209-223.

Deryugina, E.I., Ratnikov, B.I., Postnova, T.I., Rozanov, D.V., Strongin, A.Y., 2002b. Processing of integrin alpha(v) subunit by membrane type 1 matrix metalloproteinase stimulates migration of breast carcinoma cells on vitronectin and enhances tyrosine phosphorylation of focal adhesion kinase. J. Biol. Chem. 277, 9749-9756.

Deryugina, E.I., Soroceanu, L., Strongin, AY, 2002a. Up-regulation of vascular endothelial growth factor by membrane-type 1 matrix metalloproteinase stimulates human glioma xenograft growth and angiogenesis. Cancer Res. 62, 580-588.

Egeblad, M., Werb, Z., 2002. New functions for the matrix metalloproteinases in cancer progression. Nature Rev. Cancer 2, 161-174.

Galvez, B.G., Matias-Roman, S., Albar, J.P., Sanchez-Madrid, E, Arroyo, A.G., 2001. Membrane type 1-matrix metalloproteinase is activated during migration of human endothelial cells and modulates endothelial motility and matrix remodeling. J. Biol. Chem. 276, 3749137500

Gilles, C., Polette, M., Coraux, C., et al., 2001. Contribution of MT1-MMP and of human laminin-5 gamma2 chain degradation to mammary epithelial cell migration. J. Cell Sci. 114, 2967-2976.

Gingras, D., Bousquet-Gagnon, N., Langlois, S., Lachambre, M.P., Annabi, B., Beliveau, R., 2001. Activation of the extracellular signalregulated protein kinase (ERK) cascade by membrane-type-1 matrix metalloproteinase (MT1-MMP). FEBS Lett. 507, $231-236$.

Hajitou, A., Sounni, N.E., Devy, L., et al., 2001. Down-regulation of vascular endothelial growth factor by tissue inhibitor of metalloproteinase-2: effect on in vivo mammary tumor growth and angiogenesis. Cancer Res. 61, 3450-3457.

Hiraoka, N., Allen, E., Apel, I.J., Gyetko, M.R., Weiss, S.J., 1998. Matrix metalloproteinases regulate neovascularization by acting as pericellular fibrinolysins. Cell 95, 365-377.

Hofmann, U.B., Westphal, J.R., Zendman, A.J., Becker, J.C., Ruiter, D.J., van Muijen, G.N., 2000. Expression and activation of matrix metalloproteinase-2 (MMP2) and its co-localization with membrane-type 1 matrix metalloproteinase (MT1-MMP) correlate with melanoma progression. J. Pathol. 191, 245-256.

Holmbeck, K., Bianco, P., Caterina, J., et al., 1999. MT1-MMP-deficient mice develop dwarfism, osteopenia, arthritis, and connective tissue disease due to inadequate collagen turnover. Cell 99, 81-92.

Hotary, K., Allen, E., Punturieri, A., Yana, I., Weiss, S.J., 2000. Regulation of cell invasion and morphogenesis in a three-dimensional type I collagen matrix by membrane-type matrix metalloproteinases 1, 2, and 3. J. Cell Biol. 149, 1309-1323.

Itoh, Y., Takamura, A., Ito, N., et al., 2001. Homophilic complex formation of MT1-MMP facilitates proMMP2 activation on the cell surface and promotes tumor cell invasion. EMBO J. 20, 4782-4793.

Jiang, Y., Goldberg, I.D., Shi, Y.E., 2002. Complex roles of tissue inhibitors of metalloproteinases in cancer. Oncogene 21, $2245-2252$.

Kajita, M., Itoh, Y., Chiba, T., et al., 2001. Membrane-type 1 matrix metalloproteinase cleaves CD44 and promotes cell migration. J. Cell Biol. 153, 893-904.

Kolkenbrock, H., Hecker-Kia, A., Orgel, D., Ulbrich, N., Will, H., 1997. Activation of progelatinase A and progelatinase A/TIMP-2 complex by membrane type 2-matrix metalloproteinase. Biol. Chem. 378, 71-76.

Koshikawa, N., Giannelli, G., Cirulli, V., Miyazaki, K., Quaranta, V., 2000. Role of cell surface metalloprotease MT1-MMP in epithelial cell migration over laminin-5. J. Cell Biol. 148, 615-624.

Lehti, K., Lohi, J., Juntunen, M.M., Pei, D., Keski-Oja, J., 2002. Oligomerization through hemopexin and cytoplasmic domains regulates the activity and turnover of membrane-type 1 matrix metalloproteinase. J. Biol. Chem. 277, 8440-8448.

Llano, E., Pendas, A.M., Freije, J.P., et al., 1999. Identification and characterization of human MT5-MMP, a new membrane-bound activator of progelatinase a overexpressed in brain tumors. Cancer Res. 59, 2570-2576. 
Published in: Matrix Biology (2003), vol. 22, iss. 1, pp. 55-61.

Status: Postprint (Author's version)

Masson, R., Lefebvre, O., Noel, A., et al., 1998. In vivo evidence that the stromelysin-3 metalloproteinase contributes in a paracrine manner to epithelial cell malignancy. J. Cell Biol. 140, 1535-1541.

McCawley, L.J., Matrisian, L.M., 2000. Matrix metalloproteinases: multifunctional contributors to tumor progression. Mol. Med. Today 6, $149-156$

McQuibban, G.A., Butler, G.S., Gong, J.H., et al., 2001. Matrix metalloproteinase activity inactivates the CXC chemokine stromal cellderived factor-1. J. Biol. Chem. 276, 43503-43508.

Mori, M., Mimori, K., Shiraishi, T., et al., 1997. Analysis of MT1-MMP and MMP2 expression in human gastric cancers. Int. J. Cancer 74, 316-321.

Morrison, C.J., Butler, G.S., Bigg, H.F., Roberts, C.R., Soloway, P.D., Overall, C.M., 2001. Cellular activation of MMP2 (gelatinase A) by MT2-MMP occurs via a TIMP-2-independent pathway. J. Biol. Chem. 276, 47402-47410.

Mu, D., Cambier, S., Fjellbirkeland, L., et al., 2002. The integrin alpha(v)beta8 mediates epithelial homeostasis through MT1-MMPdependent activation of TGF-betal. J. Cell Biol. 157, 493-507.

Nawrocki, B., Polette, M., Marchand, V., et al., 1997. Expression of matrix metalloproteinases and their inhibitors in human bronchopulmonary carcinomas: quantificative and morphological analyses. Int. J. Cancer 72, 556-564.

Nielsen, B.S., Rank, F., Lopez, J.M., et al., 2001. Collagenase-3 expression in breast myofibroblasts as a molecular marker of transition of ductal carcinoma in situ lesions to invasive ductal carcinomas. Cancer Res. 61, 7091-7100.

Noel, A., Pauw-Gillet, M.C., Purnell, G., Nusgens, B., Lapiere, CM., Foidart, J.M., 1993. Enhancement of tumorigenicity of human breast adenocarcinoma cells in nude mice by matrigel and fibroblasts. Br. J. Cancer 68, 909-915.

Noel, A.C., Polette, M., Lewalle, J.M., et al., 1994. Coordinate enhancement of gelatinase A mRNA and activity levels in human fibroblasts in response to breast-adenocarcinoma cells. Int. J. Cancer 56, 331-336.

Okada, A., Bellocq, J.P., Rouyer, N., et al., 1995. Membrane-type matrix metalloproteinase (MT-MMP) gene is expressed in stromal cells of human colon, breast, and head and neck carcinomas. Proc. Natl. Acad. Sci. USA 92, 2730-2734.

Pei, D., 1999. Identification and characterization of the fifth membrane-type matrix metalloproteinase MT5-MMP. J. Biol. Chem. 274, 89258932.

Pei, D., Kang, T., Qi, H., 2000. Cysteine array matrix metalloproteinase (CA-MMP)/MMP23 is a type II transmembrane matrix metalloproteinase regulated by a single cleavage for both secretion and activation. J. Biol. Chem. 275, 33988-33997.

Pepper, M.S., 2001. Role of the matrix metalloproteinase and plasminogen activator-plasmin systems in angiogenesis. Arterioscler. Thromb. Vasc. Biol. 21, 1104-1117.

Polette, M., Gilles, C., Marchand, V., Seiki, M., Tournier, J.M., Birembaut, P., 1997. Induction of membrane-type matrix metalloproteinase 1 (MT1-MMP) expression in human fibroblasts by breast adenocarcinoma cells. Clin. Exp. Metastasis 15, 157-163.

Sounni, N.E., Baramova, E.N., Munaut, C., et al., 2002a. Expression of membrane type 1 matrix metalloproteinase (MT1-MMP) in A2058 melanoma cells is associated with MMP2 activation and increased tumor growth and vascularization. Int. J. Cancer 98, 23-28.

Sounni, N.E., Devy, L., Hajitou, A., et al., 2002b. MT1-MMP expression promotes tumor growth and angiogenesis through an up-regulation of vascular endothelial growth factor expression. FASEB J. 16, 555-564.

Sternlicht, M.D., Werb, Z., 2001. How matrix metalloproteinases regulate cell behavior. Annu. Rev. Cell Dev. Biol. 17, 463-516.

Takino, T., Sato, H., Shinagawa, A., Seiki, M., 1995. Identification of the second membrane-type matrix metalloproteinase (MT-MMP2) gene from a human placenta cDNA library. MT-MMPs form a unique membrane-type subclass in the MMP family. J. Biol. Chem. 270, 23013-23020.

Tsunezuka, Y., Kinoh, H., Takino, T., et al., 1996. Expression of membrane-type matrix metalloproteinase 1 (MT1-MMP) in tumor cells enhances pulmonary metastasis in an experimental metastasis assay. Cancer Res. 56, 5678-5683.

Unden, A.B., Sandstedt, B., Bruce, K., Hedblad, M., Stahle-Backdahl, M., 1996. Stromelysin-3 mRNA associated with myofibroblasts is overexpressed in aggressive basal cell carcinoma and in dermatofibroma but not in dermatofibrosarcoma. J. Invest Dermatol. 107, $147-153$.

Velasco, G., Cal, S., Merlos-Suarez, A., et al., 2000. Human MT6-matrix metalloproteinase: identification, progelatinase A activation, and expression in brain tumors. Cancer Res. 60, 877-882.

Wang, X., Pei, D., 2001. Shedding of membrane type matrix metalloproteinase 5 by a furin-type convertase: a potential mechanism for down-regulation. J. Biol. Chem. 276, 35953-35960.

Wang, Z., Juttermann, R., Soloway, P.D., 2000. TIMP-2 is required for efficient activation of proMMP2 in vivo. J. Biol. Chem. 275, 2641126415 .

Yana, I., Seiki, M., 2002. MT-MMPs play pivotal roles in cancer dissemination. Clin. Exp. Metastasis 19, 209-215.

Yu, Q., Stamenkovic, I., 1999. Localization of matrix metalloproteinase 9 to the cell surface provides a mechanism for CD44-mediated tumor invasion. Genes Dev. 13, 35-48.

Zhou, Z., Apte, S.S., Soininen, R., Cao, R., Baaklini, G.Y., Rauser, R.W., Wang, J., Cao, Y, Tryggvason, K., 2000. Impaired endochondral ossification and angiogenesis in mice deficient in membrane-type matrix metalloproteinase I. Proc. Natl. Acad. Sci. U.S.A. 11 97, 40524057. 